\title{
Impact of Rotation Correction after Brace Treatment on Prognosis in Adolescent Idiopathic Scoliosis
}

\author{
Kentaro Yamane, Tomoyuki Takigawa, Masato Tanaka, \\ Yoshihisa Sugimoto, Shinya Arataki, Toshifumi Ozaki \\ Department of Orthopaedic Surgery, Okayama University Graduate School of Medicine, Okayama, Japan
}

\begin{abstract}
Study Design: Level 4 retrospective review.
Purpose: Brace treatment is the standard nonoperative treatment for adolescent idiopathic scoliosis (AIS). Rotation correction is also important, because AIS involves a rotation deformity. The purpose of this study was to evaluate the impact of rotation correction after Osaka Medical College (OMC) brace treatment on clinical outcomes in AIS.

Overview of Literature: Brace treatment has a significant effect on the progression of AIS. However, few reports have examined rotation correction after brace treatment.

Methods: A total of 46 patients who wore the OMC brace were retrospectively reviewed. The curve magnitude was determined according to the Cobb method, and the rotation angle of the apical vertebrae was measured by the modified Nash-Moe method. Based on the difference in the rotation angle before and after the initial brace treatment, patients were divided into two groups. Group $\mathrm{A}$ $(n=33)$ was defined as no change or improvement of the rotation angle; group $B(n=13)$ was defined as deterioration of the rotation angle. If the patients had curve or rotation progression of $5^{\circ}$ or more at skeletal maturity, or had undergone surgery, the treatment was considered a failure.

Results: Differences of rotation angle between before and after the initial brace treatment were $2^{\circ} \pm 2^{\circ}$ in group $A$ and $-3^{\circ} \pm 2^{\circ}$ in group $B(p<0.001)$. The rates of treatment failure were $42 \%$ in group $A$ and $77 \%$ in group $B(p<0.05)$. This study included 25 patients with Lenke type $1(54 \%)$. Group A (24\%) with Lenke type 1 also had a significantly better success rate of brace treatment than group $\mathrm{B}(75 \%)(p<0.05)$.

Conclusions: Insufficient rotation correction increased brace treatment failure. Better rotation correction resulted in a higher success rate of brace treatment in patients with Lenke type 1.
\end{abstract}

Keywords: Adolescent idiopathic scoliosis; Brace treatment; Rotation correction; Brace treatment failure; Clinical outcome

\section{Introduction}

Brace treatment is the standard nonoperative treatment for adolescent idiopathic scoliosis (AIS). Proper wearing of a brace is effective in preventing curve progression [1-3]. Although coronal curve correction is always the focus, rotation correction is also important, because AIS involves a rotation deformity. However, few reports have examined

\footnotetext{
Received Dec 15, 2015; Revised Feb 25, 2016; Accepted Feb 26, 2016

Corresponding author: Kentaro Yamane

Department of Orthopaedic Surgery, Okayama University Graduate School of Medicine,

2-5-1, Shikata-cho, Kita-ku, Okayama 700-8558, Japan

Tel: +81-86-235-7273, Fax: +81-86-223-9727, E-mail: woodblocks0311@gmail.com
} 
rotation correction after brace treatment [4].

The Osaka Medical College (OMC) brace was prescribed for patients with adolescent scoliosis in our institute. The OMC brace is an underarm brace developed by OMC in the 1970s. The correction mechanism depends on the principle of three-point support to achieve the corrective effect on thoracic bending. An armpit pad is for correction of the high thoracic curve by the righting reflex. OMC brace treatment for AIS could decrease the progression of curves and alter the natural history $[5,6]$.
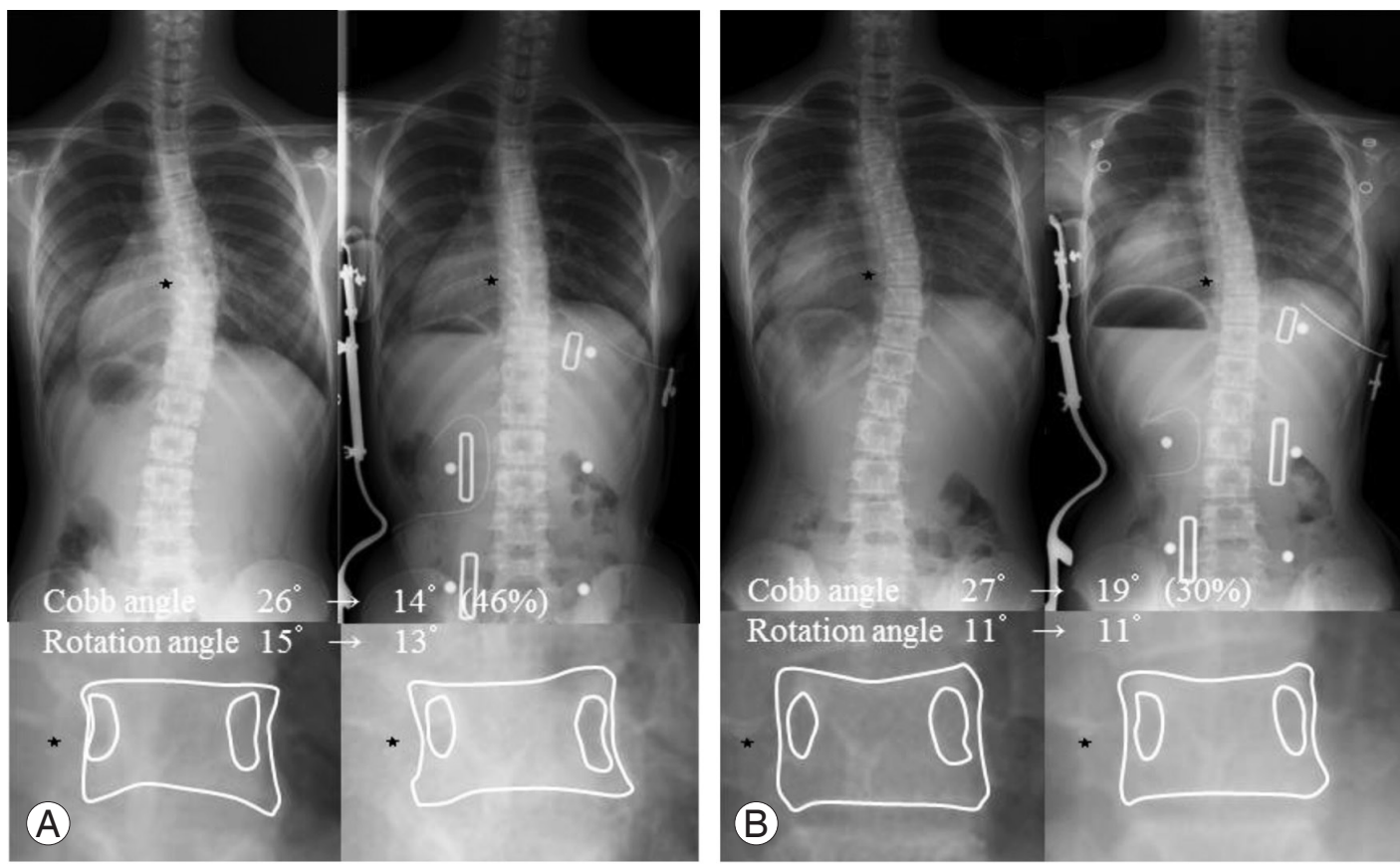

Rotation angle $11^{\circ} \rightarrow 11$

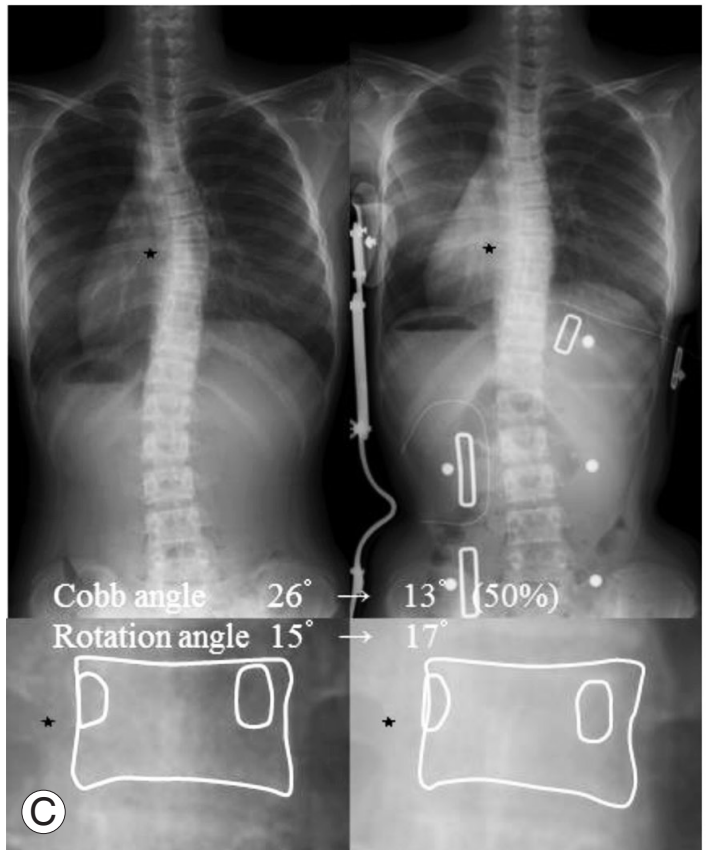

(B)
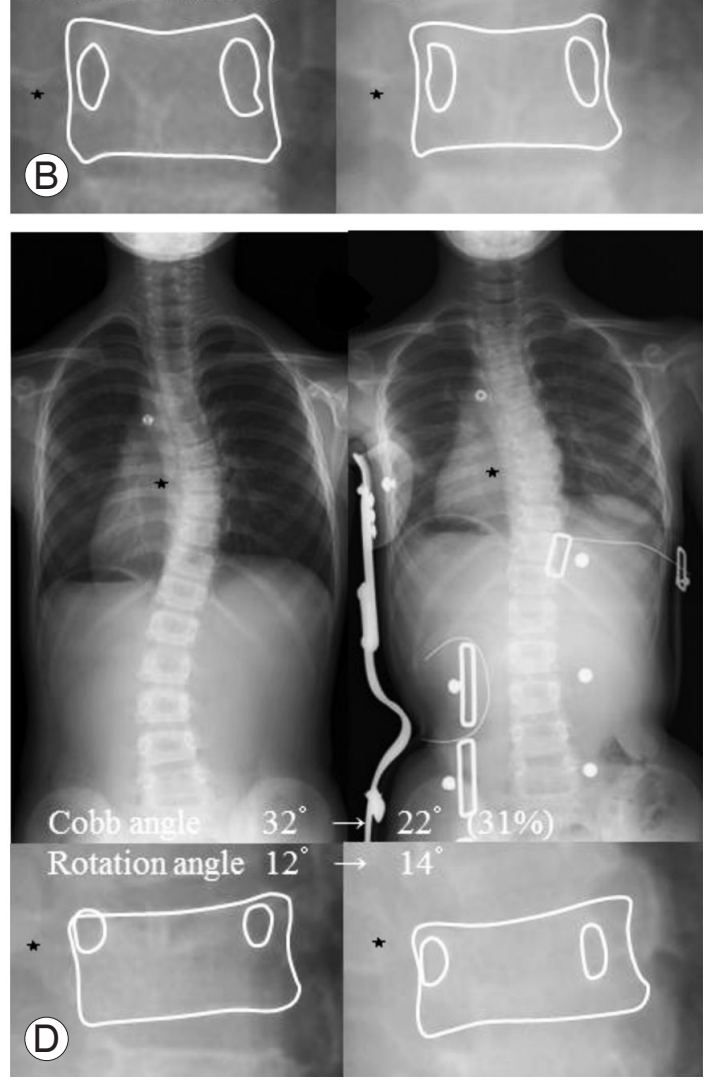

Fig. 1. Representative posteroanterior radiographs of the pre- and post-bracing treatment of the four groups. The left side shows the pre-bracing image and the right shows the post-bracing image. The * indicates the apical vertebra. (A) A patient in group A1 with good coronal and rotational correction. (B) A patient in group A2 with poor coronal and good rotational correction. (C) A patient in group B1 with good coronal and poor rotational correction. (D) A patient in group B2 with poor coronal and rotational correction. Numbers in parentheses are the correction rates of the Cobb angle. 
The purpose of this study was to evaluate the impact of rotation correction on clinical outcomes after OMC brace treatment.

\section{Materials and Methods}

The OMC brace treatment was used for AIS patients who met the following criteria: age 10 years or older, Risser stage $0-4$, Cobb angle $20^{\circ}-40^{\circ}$, and no prior treatment. Patients wore the brace for 12 or more hours per day, and that they completed brace treatment for a minimum of 12 months before evaluation and then achieved skeletal maturity. Forty-six patients met the study inclusion criteria. All radiographs for each patient were reviewed to determine the curve pattern and measurements. The curve was classified using the Lenke classification method [7]. The curve magnitude was determined according to the Cobb method and the rotation angle of the apical vertebrae was measured with the modified Nash-Moe method [8,9]. The modified Nash-Moe method approximates the vertebral rotation angle by measuring the percentage displacement of the convex pedicle with respect to the vertebral body width. One percent displacement was regarded as one degree vertebral rotation. Both the Cobb angle and the rotation angle were recorded prior to treatment, at initiation of bracing, and at the final follow-up immediately after completing brace treatment. The radiographic measurements were done on brace at the time of the start of bracing, and off brace at pre-treatment and at final followup. The improvement rate of the Cobb angle was calculated using the following formula: (Cobb angle at pretreatment-Cobb angle at initiation of bracing or at final follow-up)/Cobb angle at pre-treatmentí100\%.

Based on the difference in the rotation angle between before and after the initial brace treatment, patients were divided into two groups. Group A $(\mathrm{n}=33)$ had $0^{\circ}$ or more improvement of the rotation angle. Group B $(n=13)$ had more than $0^{\circ}$ deterioration of the rotation angle. Each group was subdivided into two groups based on the improvement rate of the Cobb angle after initial brace treatment. Group A1 ( $\mathrm{n}=16)$ and B1 $(\mathrm{n}=6)$ showed 30\% or more improvement of the Cobb angle, while group A2 $(n=17)$ and B2 $(n=7)$ showed less than $30 \%$ improvement of the Cobb angle. Representative radiographs are shown in Fig. 1. If the patients had a curve or rotation progression of $5^{\circ}$ or more at skeletal maturity or had undergone surgery, the treatment was considered a failure.
All the data are expressed as mean \pm standard deviation. Comparative analyses between the two groups were performed with the $t$-test for continuous variables and the chi-squared test for categorical variables. Among the four subdivided groups, analysis of variance (ANOVA) for continuous variables and the chi-squared test for categorical variables were used. The Tukey-Kramer multiple comparison procedure was used to identify the specific differences among the four groups after a significant ANOVA result. Residual analysis was used to identify the categories responsible for a significant chi-square statistic. A $p$-values $<0.05$ were considered significant.

\section{Results}

\section{Demographic data and comparisons of measurement parameters}

The mean brace treatment period was $27 \pm 13$ months in group A and $32 \pm 18$ months in group B. No significant differences were found in mean age, Risser stage, Cobb angle, and rotation angle before brace treatment between the two groups (Table 1). After the initial brace treatment and at the final follow-up, there were no significant differences in the Cobb angle and the rotation angle between the two groups. There were significant differences in the rotation angle from pre-treatment to initial treatment and from pre-treatment to final follow-up between the two groups (both $p<0.001$ ) (Table 1).

\section{Brace treatment failure}

The rates of treatment failure were $42 \%(14 / 33)$ in group A and 77\% (10/13) in group B (Fig. 2A); the treatment failure rate was significantly lower in group $\mathrm{A}$ than in group B $(p<0.05)$. In group A, 3 patients had both $5^{\circ}$ or more curve and rotation progression, 4 had only curve progression, 4 had only rotation progression, and 3 underwent surgery with less than $5^{\circ}$ of curve and rotation progression, while in group B, 4 patients had both $5^{\circ}$ or more curve and rotation progression, one had only curve progression, 4 had only rotation progression, and one underwent surgery with less than $5^{\circ}$ of curve and rotation progression. In patients with $5^{\circ}$ or more curve and/or rotation progression, 8 patients in group $\mathrm{A}$ and 4 patients in group B underwent surgery. 
Table 1. Demographic data and comparison of measurement parameters

\begin{tabular}{|c|c|c|c|}
\hline Characteristic & Group A & Group B & $p$-value \\
\hline No. of patients & 33 & 13 & - \\
\hline Age (yr) & $12.5 \pm 1.2$ & $12.5 \pm 1.7$ & 0.987 \\
\hline Risser grade & $2.5 \pm 1.3$ & $2.1 \pm 1.6$ & 0.358 \\
\hline Treatment period (mo) & $27.4 \pm 12.8$ & $31.6 \pm 18.0$ & 0.376 \\
\hline \multicolumn{4}{|l|}{ Cobb angle $\left(^{\circ}\right)$} \\
\hline At pre-treatment & $32 \pm 7$ & $35 \pm 7$ & 0.236 \\
\hline At initiation of bracing & $21 \pm 8$ & $24 \pm 10$ & 0.257 \\
\hline At final follow-up & $34 \pm 10$ & $42 \pm 17$ & 0.145 \\
\hline \multicolumn{4}{|c|}{ Improve rate of Cobb angle $(\%)^{\mathrm{a})}$} \\
\hline At initiation of bracing & $35 \pm 17$ & $32 \pm 21$ & 0.551 \\
\hline At final follow-up & $-6 \pm 22$ & $-20 \pm 43$ & 0.299 \\
\hline \multicolumn{4}{|l|}{ Rotation angle $\left({ }^{\circ}\right)$} \\
\hline At pre-treatment & $18 \pm 7$ & $16 \pm 6$ & 0.305 \\
\hline At initiation of bracing & $16 \pm 7$ & $19 \pm 6$ & 0.160 \\
\hline At final follow-up & $20 \pm 8$ & $23 \pm 8$ & 0.297 \\
\hline \multicolumn{4}{|c|}{ Differences of rotation angle $\left({ }^{o}\right)^{b}$} \\
\hline At initiation of bracing & $2 \pm 2$ & $-3 \pm 2$ & $<0.001$ \\
\hline At final follow-up & $-2 \pm 4$ & $-7 \pm 6$ & $<0.001$ \\
\hline
\end{tabular}

The values are given as the mean and the standard deviation.

Group $A, 0^{\circ}$ or more improvement of the rotation angle after the initial brace treatment; Group $B$, more than $0^{\circ}$ deterioration of the rotation angle after the initial brace treatment.

a)Improve rate was calculated using the following formula: (Cobb angle at pre-treatment-Cobb angle at initiation of bracing or at final follow-up)/ Cobb angle at pre-treatment $\times 100 \%$; ${ }^{b}$ Differences of rotation angle was calculated using the following formula: rotation angle at pre-treatmentrotation angle at initiation of bracing or at final follow-up.

\section{All patients}

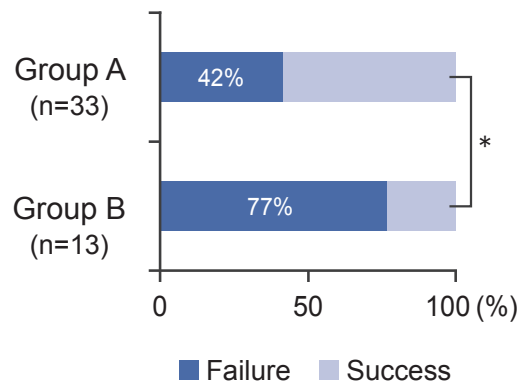

(A) Chi-square, ${ }^{*} p<0.05$ OR: 0.2

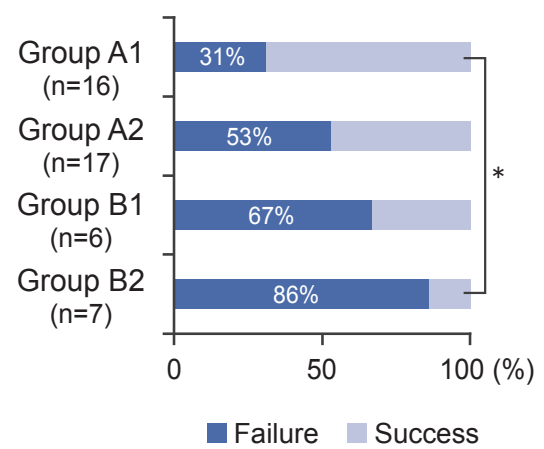

(B) Chi-square residual analysis ${ }^{*} p<0.05$

Fig. 2. Rates of treatment failure. (A) The treatment failure rate is significantly lower in group $A(14 / 33$, $42 \%)$ than in group $B(10 / 13,77 \%)$. (B) Subdivision comparison; the treatment failure rates are $31 \%(5 / 16)$ in group $A 1,53 \%(9 / 17)$ in group $A 2,67 \%(4 / 6)$ in group $B 1$, and $86 \%(6 / 7)$ in group B2. Group $A, 0^{\circ}$ or more improvement of the rotation angle after the initial brace treatment; Group $B$, more than $0^{\circ}$ deterioration of the rotation angle after the initial brace treatment; Group $A 1, B 1,30 \%$ or more improvement of Cobb angle after the initial brace treatment; Group A2, B2, less than 30\% improvement of Cobb angle after the initial brace treatment. 
Table 2. Subdivided demographic data and comparison of measurement parameters

\begin{tabular}{|c|c|c|c|c|c|}
\hline Characteristic & Group A1 & Group A2 & Group B1 & Group B2 & $p$-value \\
\hline No. of patients & 16 & 17 & 6 & 7 & - \\
\hline Age (yr) & $12.1 \pm 1.1$ & $12.9 \pm 1.2$ & $12.5 \pm 2.1$ & $12.6 \pm 1.5$ & 0.533 \\
\hline Risser grade & $2.0 \pm 1.3$ & $2.9 \pm 1.0$ & $2.2 \pm 1.7$ & $2.0 \pm 1.5$ & 0.173 \\
\hline Treatment period (mo) & $33.8 \pm 14.2$ & $21.4 \pm 7.7^{c 1}$ & $26.3 \pm 10.0$ & $36.1 \pm 22.7$ & $<0.05$ \\
\hline \multicolumn{6}{|l|}{ Cobb angle $\left(^{\circ}\right)$} \\
\hline At pre-treatment & $30 \pm 6$ & $34 \pm 8$ & $33 \pm 6$ & $36 \pm 7$ & 0.333 \\
\hline At initiation of bracing & $16 \pm 6$ & $26 \pm 6^{d)}$ & $17 \pm 5^{e)}$ & $31 \pm 8^{\mathrm{d}, \mathrm{fl}}$ & $<0.01$ \\
\hline At final follow-up & $31 \pm 10$ & $37 \pm 9$ & $39 \pm 18$ & $44 \pm 17$ & 0.111 \\
\hline \multicolumn{6}{|c|}{ Improve rate of Cobb angle $(\%)^{a)}$} \\
\hline At initiation of bracing & $48 \pm 14$ & $23 \pm 9^{\text {b) }}$ & $50 \pm 14^{9)}$ & $16 \pm 8^{\mathrm{d}, \mathrm{h})}$ & $<0.01$ \\
\hline At final follow-up & $-2 \pm 22$ & $-11 \pm 22$ & $-20 \pm 57$ & $-20 \pm 30$ & 0.451 \\
\hline \multicolumn{6}{|l|}{ Rotation angle $\left({ }^{\circ}\right)$} \\
\hline At pre-treatment & $17 \pm 7$ & $18 \pm 7$ & $12 \pm 4$ & $19 \pm 5$ & 0.193 \\
\hline At initiation of bracing & $15 \pm 6$ & $16 \pm 7$ & $15 \pm 5$ & $22 \pm 5$ & 0.102 \\
\hline At final follow-up & $19 \pm 8$ & $21 \pm 9$ & $21 \pm 10$ & $25 \pm 6$ & 0.518 \\
\hline \multicolumn{6}{|c|}{ Differences of rotation angle $\left({ }^{\circ}\right)^{\mathrm{b})}$} \\
\hline At initiation of bracing & $2 \pm 2$ & $2 \pm 2$ & $-3 \pm 2^{\mathrm{d}, \mathrm{gl}}$ & $-3 \pm 2^{d, g)}$ & $<0.01$ \\
\hline At final follow-up & $-2 \pm 3$ & $-3 \pm 4$ & $-9 \pm 8^{c, e)}$ & $-6 \pm 4$ & $<0.05$ \\
\hline
\end{tabular}

The values are given as the mean and the standard deviation.

Group $\mathrm{A} 1,0^{\circ}$ or more improvement of the rotation angle and $30 \%$ or more improvement of Cobb angle after the initial brace treatment; Group $A 2,0^{\circ}$ or more improvement of the rotation angle and less than $30 \%$ improvement of Cobb angle after the initial brace treatment; Group B1, more than $0^{\circ}$ deterioration of the rotation angle and 30\% or more improvement of Cobb angle after the initial brace treatment; Group B2, more than $0^{\circ}$ deterioration of the rotation angle and less than $30 \%$ improvement of Cobb angle after the initial brace treatment.

a)Improve rate was calculated using the following formula: (Cobb angle at pre-treatment-Cobb angle at initiation of bracing or at final follow-up)/ Cobb angle at pre-treatment $\times 100 \%$; bifferences of rotation angle was calculated using the following formula: rotation angle at pre-treatmentrotation angle at initiation of bracing or at final follow-up; ${ }^{c \mid} p<0.05$ vs. Group $\mathrm{A} 1$; ${ }^{\mathrm{d})} p<0.001$ vs. Group $\mathrm{A} 1$; ${ }^{\text {el }} p<0.05$ vs. Group $\mathrm{A} 2$; ${ }^{\text {fl }} p<0.01$ vs. Group B1; ${ }^{\text {g) }} p<0.001$ vs. Group $\mathrm{A} 2$; ${ }^{\text {h) }} p<0.001$ vs. Group B1.

\section{Subdivided comparison}

Patients with a low correction rate of the Cobb angle (group A2, B2) tended to have more treatment failures compared to those with a high correction rate of the Cobb angle (group A1, B1). The rates of treatment failure were $31 \%(5 / 16)$ in group $A 1,53 \%(9 / 17)$ in group A2, $67 \%(4 / 6)$ in group B1, and $86 \%(6 / 7)$ in group B2. The treatment failure rate was significantly lower in group A1 than in group B2 $(p<0.05)$ (Table 2, Fig. 2 B).

This study included 25 patients with Lenke type 1 (54\%), 3 with Lenke type 2 (7\%), 7 with Lenke type 3 (15\%), none with Lenke type 4 (0\%), 6 with Lenke type 5 (13\%), and 5 with Lenke type 6 (11\%) (Table 3$)$. In patients with Lenke type 1 , the rates of treatment failure were $25 \%(2 / 8)$ in group A1, 22\% (2/9) in group A2, 75\% (3/4) in group
Table 3. The details of curve type in groups

\begin{tabular}{|c|c|c|}
\hline Characteristic & Group A & Group B \\
\hline $\begin{array}{l}\text { No. of patients } \\
\text { (failure/surgery no.) }\end{array}$ & $33(14 / 11)$ & $13(10 / 6)$ \\
\hline \multicolumn{3}{|l|}{ Lenke type } \\
\hline Type 1 & $17(4 / 3)$ & $8(6 / 2)$ \\
\hline Type 2 & $3(1 / 1)$ & \\
\hline Type 3 & $3(2 / 1)$ & $4(3 / 3)$ \\
\hline Type 4 & $0(0)$ & \\
\hline Type 5 & $6(4 / 3)$ & \\
\hline Type 6 & $4(3 / 3)$ & $1(1 / 1)$ \\
\hline
\end{tabular}

Group $A, 0^{\circ}$ or more improvement of the rotation angle after the initial brace treatment; Group $B$, more than $0^{\circ}$ deterioration of the rotation angle after the initial brace treatment. 


\section{Lenke type 1}

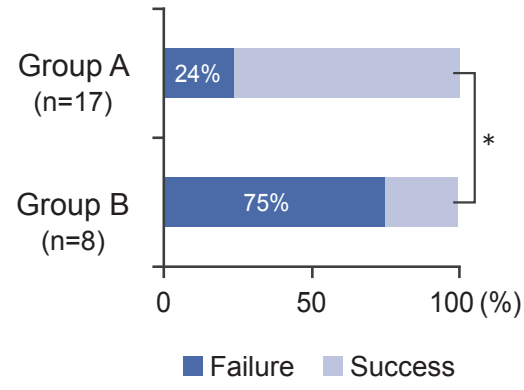

(A) Chi-square, ${ }^{*} p<0.05$ OR: 0.11

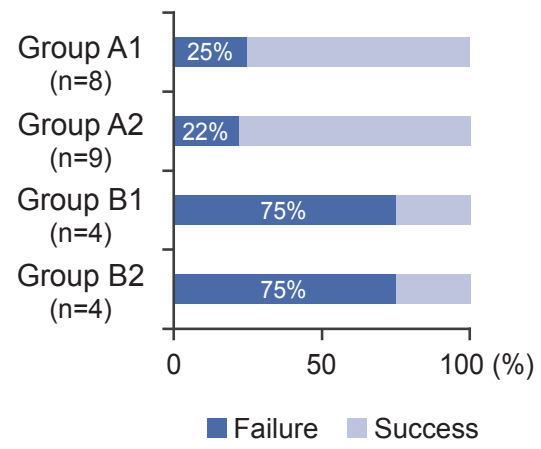

Fig. 3. The treatment failure rates of patients with Lenke type 1. (A) Group A (4/17, 24\%) has a better success rate of brace treatment than group $B(6 / 8,75 \%)$. (B) The subdivided rates of treatment failure are $25 \%(2 / 8)$ in group $A 1,22 \%(2 / 9)$ in group $A 2,75 \%(3 / 4)$ in group $B 1$, and $75 \%(3 / 4)$ in group $B 2$. Group $A, 0^{\circ}$ or more improvement of the rotation angle after the initial brace treatment; Group $B$, more than $0^{\circ}$ deterioration of the rotation angle after the initial brace treatment; Group A1, B1, 30\% or more improvement of Cobb angle after the initial brace treatment; Group A2, B2, less than 30\% improvement of Cobb angle after the initial brace treatment.

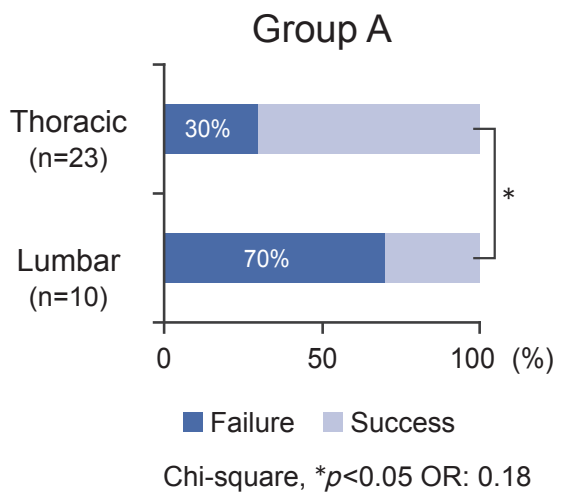

Fig. 4. Comparative analysis of thoracic and lumbar curves in group $A$. The rates of treatment failure are $30 \%(7 / 23)$ for thoracic curve and $70 \%(7 / 10)$ for lumbar curve. Thoracic, Lenke type 1-4; Lumbar, Lenke type 5, 6.

B1, and 75\% (3/4) in group B2. Group A $(4 / 17,24 \%)$ with Lenke type 1 also had a significantly better brace treatment success rate than group B $(6 / 8,75 \%)(p<0.05)$ (Fig. 3 ). In the comparative analysis of the thoracic curve (Lenke type 1-4) and the lumbar curve (Lenke type 5, 6) in group A, the rates of treatment failure were $30 \%(7 / 23)$ in the thoracic curve group and 70\% (7/10) in the lumbar curve group. The treatment failure rate was significantly lower in the thoracic curve group than in the lumbar curve group $(p<0.05)$ (Fig. 4). Five patients with a thoracic curve and 6 patients with a lumbar curve in group A underwent surgery, while 5 patients with a thoracic curve and one patient with a lumbar curve in group B underwent surgery. The mean Cobb angles at final follow-up in patients with surgery were $48^{\circ} \pm 4^{\circ}$ with a thoracic curve in group A, $41^{\circ} \pm 6^{\circ}$ with a lumbar curve in group $\mathrm{A}$, and $60^{\circ} \pm 14^{\circ}$ with a thoracic curve in group $\mathrm{B}, 40^{\circ}$ with a lumbar curve in group B.

\section{Discussion}

Various factors including curve pattern, initial curve magnitudes, age, status of menarche, skeletal maturity, and even some sagittal spinal parameters, are related to curve progression of AIS [4,10-12]. The only goal of nonoperative treatment during adolescence is to prevent curve progression. Brace treatment is the most standardized nonoperative treatment. There are many reports about brace treatment, and the weight of evidence is in favor of bracing [1-3,13]. A recent randomized cohort trial also emphasized the significant effect of brace treatment on the progression of AIS until the patient reaches skeletal maturity, with a dose-response relationship reported between brace wear and outcome [14].

The failure rate of brace treatment has varied widely in previous reports, reflecting the different research conditions $[2,15,16]$. However, the cause of brace treatment failure was also related to the patient's initial condition before brace treatment, such as curve magnitudes, age, and skeletal maturity, and patient compliance after brace treat- 
ment. As another failure factor after brace treatment, we focused on the impact of rotation correction after brace treatment on the clinical outcome in the present study.

Scoliosis is composed of a rotation deformity and coronal spinal curvature [1]. Correction of both the rotation deformity and the coronal curve is essential to approximate a normal posture. Curve and rotation correction in brace treatment is usually accomplished by applying pressure posterolaterally to the major curve. The correction force is divided into two pressure forces: pressure forward and pressure laterally. Pressure forward improves the rotation deformity, and pressure laterally improves the coronal curve. By means of proper external forces, near normal contours and alignment of the spine are restored during wearing of the brace. If an adequate external force is not applied, it can lead to the deterioration of the rotation deformity despite coronal curve correction. The best correction of the Cobb angle does not always coincide with the best correction of vertebral rotation [4]. The patients in group B probably received backward pressure force by applying pressure anterolaterally to the major curve, instead of adequate forward pressure. This might lead to the deterioration of only the rotation angle. The importance of vertebral rotation correction with brace treatment is not well established. Vertebral rotation deformity before brace treatment was reported to be correlated with the risk of requiring surgery, but not with the chance of curve control [17]. Reduction of both vertebral rotation and the Cobb angle after brace treatment is reportedly a prognostic indicator for a good outcome [4]. If the patient wears a brace for longer hours with an inadequate rotational state, it may lead to an unsatisfactory result.

This study suggests that insufficient rotation correction increased brace treatment failure when the results were compared between groups A and B (Fig. 2A). In the subdivision comparison of group A, patients with a low Cobb angle correction rate (group A2; 53\%) tended to develop more treatment failures than those with a high Cobb angle correction rate (group A1; 31\%) (Fig. 2B). However, this tendency was highly related to the effect of curve type. Group A2 contained more patients with a lumbar curve (Lenke type 5,6). Curve type analysis (Fig. 4) showed that the treatment failure rate was significantly higher in the lumbar curve group than in the thoracic curve group. The surgical indication for the Cobb angle differed between the thoracic curve and the lumbar curve. Our surgical indication according to Cobb angle was $45^{\circ}-50^{\circ}$ for the thoracic curve and $40^{\circ}-45^{\circ}$ for the lumbar curve. Larger curves beyond these ranges reportedly progress even after skeletal maturity [18-20]. According to our surgical indications, lumbar scoliosis cases tended to undergo surgery with a lower Cobb angle than thoracic scoliosis cases; the mean Cobb angles at final follow-up were $41^{\circ} \pm 6^{\circ}$ and $48^{\circ} \pm 4^{\circ}$ for the lumbar curve and thoracic curve, respectively. The high failure rate in the lumbar curve group seemed to be related to this different surgical indication. Focusing only on Lenke type 1, better rotation correction resulted in a higher success rate of brace treatment, regardless of the Cobb angle correction rate (Fig. 3B). Rotation correction can affect prognosis more than Cobb angle correction with Lenke type 1 . We need to ensure that we consider not only coronal curve correction, but also rotation correction of the apical vertebra in brace treatment of AIS. If the rotation correction is insufficient after initial wear, the brace should be modified quickly by changing the pressure posterolaterally to the major curve. The optimum compression site should be determined by checking radiographs.

Although the present study showed the importance of rotation correction after brace treatment, some limitations were present. First, the study was retrospective and the number of patients was relatively small, except for Lenke type 1. No conclusions can be made about curve types other than Lenke type 1 in this study. Second, the brace treatment criteria did not fully satisfy the SRS recommended criteria with respect to Risser grade [10]. Third, the modified Nash-Moe method of angle determination only provided a rough approximation of axial rotation. Computed tomography gives the most exact information to measure the rotation angle [8]. However, because of radiation exposure, it is difficult to perform computed tomography for all patients. Despite these limitations, we believe that this study has important ramifications for clinical practice.

\section{Conclusions}

Insufficient rotation correction when a brace was first applied increased brace treatment failure. Better rotation correction in brace treatment resulted in a higher brace treatment success rate in patients with Lenke type 1. On the contrary, correction of Cobb angle after bracing was less effective than rotation correction in determining success of brace treatment. 


\section{Conflict of Interest}

No potential conflict of interest relevant to this article was reported.

\section{Acknowledgments}

This work was partially supported by JSPS KAKENHI Grant Number 25861320.

\section{References}

1. Weinstein SL, Dolan LA, Cheng JC, Danielsson A, Morcuende JA. Adolescent idiopathic scoliosis. Lancet 2008;371:1527-37.

2. Dolan LA, Weinstein SL. Surgical rates after observation and bracing for adolescent idiopathic scoliosis: an evidence-based review. Spine (Phila Pa 1976) 2007; 32(19 Suppl):S91-100.

3. Lenssinck ML, Frijlink AC, Berger MY, Bierman-Zeinstra SM, Verkerk K, Verhagen AP. Effect of bracing and other conservative interventions in the treatment of idiopathic scoliosis in adolescents: a systematic review of clinical trials. Phys Ther 2005;85:1329-39.

4. Upadhyay SS, Nelson IW, Ho EK, Hsu LC, Leong JC. New prognostic factors to predict the final outcome of brace treatment in adolescent idiopathic scoliosis. Spine (Phila Pa 1976) 1995;20:537-45.

5. Kuroki H, Inomata N, Hamanaka H, Higa K, Chosa E, Tajima N. Predictive factors of Osaka Medical College $(\mathrm{OMC})$ brace treatment in patients with adolescent idiopathic scoliosis. Scoliosis 2015;10:11.

6. Kuroki H, Inomata N, Hamanaka H, Higa K, Chosa E, Tajima N. Efficacy of the Osaka Medical College (OMC) brace in the treatment of adolescent idiopathic scoliosis following Scoliosis Research Society brace studies criteria. Scoliosis 2015;10:12.

7. Lenke LG, Betz RR, Harms J, et al. Adolescent idiopathic scoliosis: a new classification to determine extent of spinal arthrodesis. J Bone Joint Surg Am 2001; 83:1169-81.

8. Lam GC, Hill DL, Le LH, Raso JV, Lou EH. Vertebral rotation measurement: a summary and comparison of common radiographic and CT methods. Scoliosis 2008;3:16.
9. Nash CL Jr, Moe JH. A study of vertebral rotation. J Bone Joint Surg Am 1969;51:223-9.

10. Richards BS, Bernstein RM, D'Amato CR, Thompson GH. Standardization of criteria for adolescent idiopathic scoliosis brace studies: SRS Committee on Bracing and Nonoperative Management. Spine (Phila Pa 1976) 2005;30:2068-75.

11. Stokes OM, Luk KD. The current status of bracing for patients with adolescent idiopathic scoliosis. Bone Joint J 2013;95B:1308-16.

12. Guo J, Liu Z, Lv F, et al. Pelvic tilt and trunk inclination: new predictive factors in curve progression during the Milwaukee bracing for adolescent idiopathic scoliosis. Eur Spine J 2012;21:2050-8.

13. Katz DE, Herring JA, Browne RH, Kelly DM, Birch JG. Brace wear control of curve progression in adolescent idiopathic scoliosis. J Bone Joint Surg Am 2010;92:1343-52.

14. Weinstein SL, Dolan LA, Wright JG, Dobbs MB. Effects of bracing in adolescents with idiopathic scoliosis. N Engl J Med 2013;369:1512-21.

15. Danielsson AJ, Hasserius R, Ohlin A, Nachemson AL. A prospective study of brace treatment versus observation alone in adolescent idiopathic scoliosis: a follow-up mean of 16 years after maturity. Spine (Phila Pa 1976) 2007;32:2198-207.

16. Janicki JA, Poe-Kochert C, Armstrong DG, Thompson GH. A comparison of the thoracolumbosacral orthoses and providence orthosis in the treatment of adolescent idiopathic scoliosis: results using the new SRS inclusion and assessment criteria for bracing studies. J Pediatr Orthop 2007;27:369-74.

17. Emans JB, Kaelin A, Bancel P, Hall JE, Miller ME. The Boston bracing system for idiopathic scoliosis. Follow-up results in 295 patients. Spine (Phila Pa 1976) 1986;11:792-801.

18. Weinstein SL, Ponseti IV. Curve progression in idiopathic scoliosis. J Bone Joint Surg Am 1983;65:44755.

19. Ascani E, Bartolozzi P, Logroscino CA, et al. Natural history of untreated idiopathic scoliosis after skeletal maturity. Spine (Phila Pa 1976) 1986;11:784-9.

20. Edgar MA. The natural history of unfused scoliosis. Orthopedics 1987;10:931-9. 